ORIGINAL ARTICLE

PRACA ORYGINALNA

\title{
SOCIAL AND EMOTIONAL INTELLIGENCE AS A BASIS FOR COMMUNICATIVE RESOURCE FORMATION IN FAMILY CAREGIVERS OF PATIENTS WITH ENDOGENOUS MENTAL DISORDERS
}

D0I: 10.36740/WLek202001121

\author{
Anna 0. Kaminska, Nataliia G. Pshuk, Yuliana Y. Martynova \\ NATIONAL PIROGOV MEMORIAL MEDICAL UNIVERSITY, VINNYTSIA, UKRAINE
}

\begin{abstract}
The aim of our study was to determine features of social and emotional intelligence in family caregivers of patients with endogenous mental disorders as a basis for communicative resource formation in family where a patient lives.

Materials and methods: A total of 273 family caregivers of patients with paranoid schizophrenia and bipolar disorder were involved into this survey under informed consent conditions. Control group included 55 mentally healthy respondents, in whose families there is no mentally sick family member. Emotional intelligence of family caregivers was measured using the psychodiagnostic test "EQ" by N. Hall. To assess level of social intelligence the J. Gilford and M. Sullivan test (in adaptation done by Mikhailova E.S.) was used. Values of $p<0.05$ were considered significant.

Results: The study revealed that family caregivers of patients with schizophrenia and affective disorders demonstrate a decrease in emotional and social intelligence indicators, which creates significant obstacles for effective interpersonal family communication and for the harmonious functioning of a family, in which a mentally sick patient lives, in general. Difficulties of emotional regulation, emotional management, recognition of emotional states of other participants of communication related to the level of emotional and social intelligence of $\mathrm{FC}$ are factors, that complicate interpersonal relations in families of patients and reduce possibilities for psychosocial adaptation of all family members. Conclusions: Revealed features should be taken into consideration when creating appropriate psycho-educational and psycho-corrective programs for family caregivers of patients with endogenous mental disorders.
\end{abstract}

KEY WORDS: family caregivers; endogenous mental disorders; coping behavior, psychocorrection

Wiad Lek. 2020;73(1):107-112

\section{INTRODUCTION}

Interpersonal communication in families of patients with endogenous mental disorders (EMD) provides multiple tasks i.e. information exchange, coordination of efforts, aimed at treatment and social rehabilitation, effective redistribution of roles in family activities; ensures development of interpersonal relationships in changed family situation, better understanding of partner and self-understanding. Prerequisites for effective interpersonal communication in a family, where patient with EMD lives, include general principles of successful family communication organization, as well as communicative resource of communicative partners: activity and openness, sufficient self-disclosure in process of communication, congruence of communication; accuracy of non-verbal communication, consistency of verbal and non-verbal messages; sensitivity to partners pronouncements, ability to listen actively and to provide appropriate feedback; impartiality and empathy in accepting a mentally sick family member as a communicative partner; creating an atmosphere of psychological security; affiliation, mutual empathy and social support, respect for the partner, which is especially important under stress, frustration and problem conditions.
Family interpersonal communication is associated with significant emotional tension and intensity of interaction [1]. Family of a mentally sick patient functions in reality, which varies according to the dynamics of his mental condition, which requires constant adaptation and communicative flexibility from family caregivers in order to maintain family homeostasis and functionality of the entire family system [2]. At the same time, due to lack of proper training, insufficient knowledge of disease and psychological distress, caused by abrupt changes in mental status of a family member, family caregivers face significant strain, now widely known as "family burden", which negatively affects communication in whole family system. Disruption of interpersonal communication is one of the most pressuring problems of family functioning in families where a patient with EMD lives [3, 4, 5, 6].

Within the last two decades, the important direction in development of psychosocial treatment for endogenous mental disorders is the emphasis on positive effects of family participation in process of therapy [7]. Psychosocial interventions are introduced into system of therapeutic measures for mentally sick patients and are considered along with psychopharmacological influences as those 
that significantly affect the final outcome of treatment in both inpatient and outpatient care [8]. Providing effective and flexible interpersonal communication in families of patients with EMD is a basis for ensuring the effectiveness of psychosocial therapy, and for psychoprophylaxis of mental disadaptation in family caregivers of patients with EMD.

Communicative resources (CR) are the basis, that not only provides effective coping with problem situations, but also enhances psychosocial adaptation. It is due to communicative psychological resources that an adaptive or non-adaptive communicative behavioral style is formed, which is based, first of all, on empathy, affiliation, locus of control, self-assessment of the individual. In a situation where the disease, on one hand, changes personality structure of a patient and leads to leveling and suppression of emotional sphere, and on the other hand - complicates adequate interpersonal communication both in family, and in society in general, study of basis for the main communicative resources in family caregivers of patients with EMD is the urgent problem. It is common knowledge that appearance of a mentally sick person in a family negatively affects quality of life of patient and his relatives. It results in changes of interpersonal relationships, states of high irritability and unmotivated aggression, or, on the contrary, states of very low energy potential of person with limited functional (emotional and volitional) capabilities. All these factors lead to disruption of psychological compensatory-adaptive reactions and conditions in relatives of patients with EMD, that creates prerequisites for psychosocial disadaptation in family caregivers $[9,10]$. The extent to which family members are able to form an adequate relationship and adapt to new living conditions depends on their personal communicative resource, which is formed on the basis of their emotional and social intelligence. Low level of communicative resource leads to passive maladaptive behavior, social isolation and disintegration of personality.

In overcoming stressful situations and performing effective interpersonal communication in families where patients with EMD live, features of emotional and social intelligence play a decisive role. Thanks to the ability to recognize and understand own emotional states and emotional states of other people, the basis for providing effective and adaptive emotional and behavioral responses in a family-friendly environment occurs.

\section{THE AIM}

The aim of our study was to determine the features of social and emotional intelligence in family caregivers of patients with EMD as a basis for communicative resource formation in family where a patient with EMD lives.

\section{MATERIALS AND METHODS}

To achieve this aim, according to the principles of bioethics and medical ethics, under the informed consent, 243 family caregivers (FC) of patients, who were diagnosed with paranoid schizophrenia (PSch) - 168 persons (main group of family caregivers of patients with PSch, MGPSch) and patients with affective disorders (AD) - bipolar disorder, recurrent depressive disorder - 75 persons (main group of family caregivers of patients with $\mathrm{AD}, \mathrm{MGAD}$ ), were examined. The inclusion criteria were: informed consent for filling up the questionnaire, clinical and psychological examination and psychological testing, absence of previous appeals to a psychiatrist or narcologist, absence of craniocerebral trauma, 1-2 degree of affinity to a mentally sick family member. There were 49 wives, 25 husbands, 94 mothers/fathers of patients in MGPSch. There were 20 wives, 25 husbands, 30 - one of the parents of patients with $\mathrm{AD}$. The subjects were in the age range from 26 to 63 years (average age: "wife" $37.5 \pm 0.8$ years, "husband" $42.3 \pm 0.9$ and "one of the parents" $60.3 \pm 3.7$ years). Most of the relatives $(64.5 \%)$ had a sufficient educational qualification (secondary and higher education), $55.1 \%$ had a permanent job. $70.4 \%$ of the respondents of MGPSch and MGAD rated their living conditions as satisfactory, while $23.1 \%$ indicated an unsatisfactory financial state of family, which changed after the appearance of a patient with a mental disorder in family. Analysis of family relationships showed that presence of adequate relationships was stated by $19.5 \%$ of the MGPSch and $28.6 \%$ of the MGAD respondents, while $46.2 \%$ of MGPSch and $26.4 \%$ of MGAD respondents described their family relationships as conflict and emotionally unstable. Control group (CG) included 55 mentally healthy persons ( 35 female and 20 male), in whose families there was no mentally ill patient and who never sought help from a psychiatrist.

Emotional intelligence of family caregivers was measured using the psychodiagnostic test "EQ" by N. Hall. This questionnaire was designed to identify the ability to understand the interpersonal relationships that are represented in emotions, and to manage emotional sphere on the basis of decision making.

Hall's emotional intelligence test defines both general level of emotional intelligence and its particular determinants (emotional awareness, ability to control emotions, self-motivation, empathy, recognition of emotional states of other people). For the examination of FC of patients with EMD a full version of test was used. Obtained results were evaluated in 5 subtests, and composite score was also taken into account.

To assess the level of social intelligence the J. Gilford and M. Sullivan test (in adaptation done by Mikhailova E.S.) was used. Study of social intelligence through social intellect as a factor of psychosocial adaptation allows to estimate ability to understand and predict behavior of people in different life situations, to recognize the intentions, feelings and emotional states of person by verbal and nonverbal expression. This test defines both general level of social intelligence and its separate components (ability to predict consequences of behavior, to understand verbal and nonverbal behavior, to understand general picture of interpersonal interaction). Processing of the received data was performed by methods of mathematical statistics. 
Table I. Indicators of emotional intelligence level in family caregivers of patients with endogenous mental disorders (mean score, $\mathrm{M} \pm \mathrm{m}$ )

\begin{tabular}{cccc}
\hline Indicators & \multicolumn{3}{c}{ Mean score (M \pm m) } \\
\cline { 2 - 4 } & MGPSch & MGAD & $14,5 \pm 1,5$ \\
\hline Emotional awareness & $7,9 \pm 2,5^{* *}$ & $12,5 \pm 1,5^{* *}$ & $15,5 \pm 1,5$ \\
\hline Managing emotions & $3,2 \pm 1,7^{* *}$ & $4,5 \pm 1,5^{* *}$ & $11,4 \pm 1,9^{* *}$ \\
\hline Self-motivation & $8,7 \pm 1,9^{* *}$ & $10,0 \pm 2,6^{*}$ & $12,0 \pm 2,6$ \\
\hline Empathy & $5,5 \pm 2,3^{*}$ & 1,9 \\
\hline Recognition of emotions of others & $5,6 \pm 1,2^{*}$ & $12,3 \pm 1,5^{*}$ & $16,3 \pm 1,5$ \\
\hline Composite score & $36,4 \pm 2,9^{* *}$ & $43,2 \pm 1,7^{* *}$ & $70,5 \pm 3,7$ \\
\hline
\end{tabular}

Note: * - data are statistically reliable $(\mathrm{P}<0,05)$; $^{* *}$ - $(\mathrm{P}<0,001)$

Table II. Indicators of emotional intelligence level in family caregivers of patients with paranoid schizophrenia depending on the duration of the disease in a mentally ill family member (mean score, $M \pm m$ )

\begin{tabular}{cccc}
\hline Indicators & \multicolumn{3}{c}{ Mean score (M \pm m) } \\
\cline { 2 - 5 } & $\begin{array}{c}\text { MGPSch1 } \\
\text { (under 4 years) }\end{array}$ & $\begin{array}{c}\text { MGPSch2 } \\
\text { (5-8 years) }\end{array}$ & $\begin{array}{c}\text { MGPSch3 } \\
(\mathbf{8}-12 \text { years) }\end{array}$ \\
\hline Emotional awareness & $8,3 \pm 1,4^{* *}$ & $9,5 \pm 1,5$ & $6,5 \pm 2,5$ \\
\hline Managing emotions & $5,2 \pm 1,9$ & $3,5 \pm 1,5$ & $-8,5 \pm 1,5^{* *}$ \\
\hline Self-motivation & $16,7 \pm 2,1^{*}$ & $10,2 \pm 1,9$ & $10,4 \pm 1,9$ \\
\hline Empathy & $15,5 \pm 2,0^{*}$ & $10,0 \pm 2,6$ & $-1,0 \pm 2,6^{*}$ \\
\hline Recognition of emotions of others & $5,6 \pm 1,1^{*}$ & $15,3 \pm 3,5^{*}$ & $-6,3 \pm 1,5$ \\
\hline Composite score & $49,4 \pm 2,5^{* *}$ & $33,2 \pm 2,7^{* *}$ & $20,5 \pm 12,8^{* *}$ \\
\hline
\end{tabular}

Note: * - data are statistically reliable $(\mathrm{P}<0,05)$; $^{* *}-(\mathrm{P}<0,001)$

\section{RESULTS AND DISCUSSION}

One of the basic determinants of interpersonal interaction is emotional intelligence as a complex integral characteristic that provides recognition of own emotions and emotions of other people, as well as creates preconditions for managing emotions in situations of interpersonal communication.

Study of the emotional intelligence level in FC of patients with EMD (Table I) made it possible to find out some differences in main groups compared to CG. Received digital data by the scale of emotional awareness (MGPSch 7.9 \pm 2.5 and MGAD $12.5 \pm 1.5$; CG $14.5 \pm 1.5 ; \mathrm{P}<0.001)$ suggest that family caregivers of patients with EMD are not always able to understand the meaning of both negative and positive emotions to gain knowledge about which decision is better to accept, that reflects reduced ability to perceive their own negative emotions as a source of understanding the need for changes in their lives, inability to feel and observe changes in their emotional states, to understand occurance of negative emotions as important indicator of life problems. They often experience significant intrapersonal difficulties due to diminished understanding of meaning of emotions for maintaining optimal well-being and control over life.

According to the emotional management subscale (MGPSch 3,2 $\pm 1,7$ and MGAD 4,5 $\pm 1,5$, CG 15,5 $\pm 1,5, \mathrm{P}$ $<0,001)$, the data indicate that respondents from both main groups compared to CG respondents, have significantly lower ability to keep calm and self-control under conditions of environmental pressure, to monitor their own feelings, as well as limited ability to cope with their feelings, when something frustrating happens; they are not entangled in negative emotions, quickly calmed down after unexpected complaints, easy to disconnect from negative experience. Thus, obtained data testify to decreased possibilities of emotional coping in presence of endogenous mental disorder in a family member of main groups respondents. Indicators of the self-motivation subscale (MGPSch $8.7 \pm$ 1.9 and MGAD $11.4 \pm 1.9$, CG $13.4 \pm 1.9, \mathrm{P}<0.001)$ reflect decreased ability to remain calm and lumped if necessary, acting in accordance with the requirements of life in FC of patients with EMD. Unlike the respondents of the CG, they can not deliberately experience a wide range of positive emotions - such as positive mood, joy, inner growth and humor, creatively solve actual problems in spite of life obstacles, easily immerse in a state of tranquility, readiness and concentration by rejecting negative feelings when it comes to acting.

Empathy is an important resource to overcome problem and emotionally-stressful situations. Good empathy skills, based on altruistic motivation, can help individuals to overcome their own stress, and help in overcoming distress to others, who are in need of empathy. Underdevelopment of empathy skills contributes to formation of selfish motivation in an emotional response. Such motivation is aimed, first of all, at preserving own well-being and reducing own emotional stress $[8,9]$. In family caregivers, in whose families a patient with EMD lives, the obtained results on the subscale of empathy (MGPSch $5.5 \pm 2.3$ and MGAD 
Table III. Indicators of emotional intelligence level in family caregivers

of patients with affective disorders depending on the duration of the disease in a mentally ill family member (mean score, $M \pm m$ )

\begin{tabular}{|c|c|c|c|}
\hline \multirow[b]{2}{*}{ Indicators } & \multicolumn{3}{|c|}{ Mean score $(\mathbf{M} \pm \mathbf{m})$} \\
\hline & $\begin{array}{c}\text { MGAD1 } \\
\text { (under } 4 \text { years) }\end{array}$ & $\begin{array}{c}\text { MGAD } 2 \\
\text { (5-8 years) }\end{array}$ & $\begin{array}{c}\text { MGAD } 3 \\
\text { (8-12 years) }\end{array}$ \\
\hline Emotional awareness & $2,3 \pm 3,4^{* *}$ & $9,5 \pm 7,5$ & $12,3 \pm 4,5^{* *}$ \\
\hline Managing emotions & $15,2 \pm 1,9$ & $12,5 \pm 1,5$ & $4,5 \pm 1,5^{*}$ \\
\hline Self-motivation & $15,7 \pm 2,1$ & $14,2 \pm 1,9$ & $11,4 \pm 1,9$ \\
\hline Empathy & $15,5 \pm 4,5$ & $20,0 \pm 2,6^{*}$ & $12,0 \pm 2,6$ \\
\hline Recognition of emotions of others & $15,6 \pm 1,1$ & $15,3 \pm 3,5$ & $12,3 \pm 1,5$ \\
\hline Composite score & $39,4 \pm 3,5$ & $43,2 \pm 4,7$ & $40,2 \pm 2,7$ \\
\hline
\end{tabular}

Note: ${ }^{*}$ - data are statistically reliable $(\mathrm{P}<0,05) ;{ }^{* *}-(\mathrm{P}<0,001)$

Table IV. Indicators of social intelligence level in family caregivers of patients with endogenous mental disorders (mean score, $M \pm m$ )

\begin{tabular}{cccccc}
\hline Group & Subtest 1 & Subtest 2 & Subtest 3 & Subtest 4 & Composite indicator \\
\hline MGPSCh $(n=168)$ & $3,22 \pm 0,5^{*}$ & $2,97 \pm 1,1^{* *}$ & $2,81 \pm 0,3^{* *}$ & $3,12 \pm 0,7^{* *}$ & $3,30 \pm 2,6^{* *}$ \\
\hline $\operatorname{MGAD}(n=75)$ & $3,49 \pm 0,9^{*}$ & $3,14 \pm 1,6^{* *}$ & $3,08 \pm 1,1^{* *}$ & $3,47 \pm 0,5^{* *}$ & $3,66 \pm 0,2^{* *}$ \\
\hline CG $(n=55)$ & $4,08 \pm 0,5$ & $4,18 \pm 0,1$ & $4,13 \pm 0,7$ & $4,49 \pm 0,2$ & $4,43 \pm 0,6$ \\
\hline
\end{tabular}

Note: * - data are statistically reliable $(\mathrm{P}<0,05)$; ** $-(\mathrm{P}<0,001)$

Table V. Indicators of social intelligence level in family caregivers of patients with endogenous mental disorder, depending on the duration of the disease (mean score, $\mathrm{M} \pm \mathrm{m}$ )

\begin{tabular}{cccccc}
\hline Group & ST1 & ST 2 & ST 3 & ST 4 & CI \\
\hline MGPSch1 $(n=57)$ & $3,12 \pm 0,8^{*}$ & $3,1 \pm 1,1^{*}$ & $2,9 \pm 0,8^{*}$ & $3,45 \pm 0,9^{*}$ & $3,30 \pm 2,6^{* *}$ \\
\hline MGPSch 2 $(n=58)$ & $2,72 \pm 0,5$ & $2,7 \pm 1,5$ & $2,41 \pm 1,3$ & $3,12 \pm 0,5$ & $2,40 \pm 1,3$ \\
\hline MGPSch 3 $(n=53)$ & $2,6 \pm 0,6$ & $2,2 \pm 1,0$ & $2,11 \pm 0,5$ & $2,82 \pm 0,7^{*}$ & $2,44 \pm 1,1$ \\
\hline MGAD1 $(n=34)$ & $3,41 \pm 0,9$ & $3,12 \pm 1,6^{*}$ & $2,5 \pm 1,3^{* *}$ & $2,87 \pm 0,5$ & $2,74 \pm 0,8$ \\
\hline MGAD 2 $(n=22)$ & $3,05 \pm 0,6$ & $2,35 \pm 1,2$ & $3,08 \pm 1,1^{* *}$ & $3,37 \pm 0,6^{* *}$ & $3,12 \pm 0,2^{* *}$ \\
\hline MGAD 3 $(n=19)$ & $2,15 \pm 1,0^{*}$ & $2,09 \pm 1,0$ & $2,1 \pm 1,5^{* *}$ & $2,12 \pm 0,5$ & $2,1 \pm 0,5^{*}$ \\
\hline CG $(n=55)$ & $4,08 \pm 0,5$ & $4,18 \pm 0,1$ & $4,13 \pm 0,7$ & $4,49 \pm 0,2$ & $4,43 \pm 0,6$ \\
\hline
\end{tabular}

Note: ${ }^{*}$ - data are statistically reliable $(\mathrm{P}<0,05) ;{ }^{* *}-(\mathrm{P}<0,001)$

$10.0 \pm 2.6, \mathrm{CG} 12.0 \pm 2.6, \mathrm{P}<0.05)$ show reduced ability to listen to the problems of other people and low sensitivity to emotional needs of others, limited understanding of emotions of the interlocutor, especially if they are not expressed directly, deteriorated recognition of emotions expressed by non-verbal means, low ability to feel the needs of the communicative partner, inability to adjust to other peoples emotions.

Indicators on the subscale of understanding the emotions of other people (MGPSch 5.6 \pm 1.2 and MGAD 12.3 \pm 1.5 , CG $16.3 \pm 1.5, \mathrm{P}<0.05)$ show that $\mathrm{FC}$ of patients with paranoid schizophrenia and affective disorders, have diminished ability to calm the communicative partner, to respond adequately to the mood, intentions and desires of others, to understand the feelings of others, to be good advisers in person-to-person relationships, to help others use their intentions to achieve personal goals. Composite evaluation (MGPSch $36.4 \pm 2.9$ and MGAD $43.2 \pm 1.7$, CG $70.5 \pm 3.7, \mathrm{P}<0.001)$ suggests that, in general, family caregivers of patients with EMD have both decreased ability to recognition of their own emotions and emotions of others, and limited ability to manage their emotions in situations of interpersonal interaction.

Unlike general intelligence, emotional and social intelligence are metacognitive abilities aimed at integrating cognitive, interpersonal and emotional information for a holistic understanding of reality, and may change under the influence of psychosocial experience. While studying the dynamics of emotional intelligence scores in family caregivers of patients with EMD, the following patterns were found (Table II): family caregivers of patients with PSch are characterized with a decline in parameters of empathy (MGPSch1 15.5 \pm 2.0 ; MGPSch2 $10.0 \pm 2$, 6; MGPSch3 $(-1,0) \pm 2,6$; P <0,001), ability to recognize emotions of other people (MGPSch1 5,6 $\pm 1,1$; MGPSch2 $15,3 \pm 3,5 ;$ MGPSch3 $(-6,3) \pm 1.5, \mathrm{P}<0.001)$, emotional control (MGPSch1 5.2 \pm 1.9, MGPSch2 $3.5 \pm 1.5$, MGPSch3 $(-8.5) \pm 1.5, \mathrm{P}<0.001)$ and composite score of emotional intelligence (MGPSch1 49.4 \pm 2.5; MGPSch2 $33.2 \pm 2.7$; MGPSch3 $20.5 \pm 12.8$; P <0.001) with duration of the disease in mentally ill family member. Such indicators can be interpreted as possible predictors of interpersonal 
communicative deviations in families of patients with EMD, which may be explained by contradictions in verbal and nonverbal communication; communicative barriers; violation and distortion of emotional exchange; inability to tolerate negative emotions on the background of lack of empathy and emotional competence.

When comparing the emotional intelligence scores in family caregivers of patients with $\mathrm{AD}$ (Table III), higher rates of emotional awareness in FC group of patients with a duration of disorder from 8 to 12 years were revealed in comparison with respondents from groups with a shorter duration of disease in a family member (MGAD1 $2.3 \pm 3.4$, MGAD2 9.5 \pm 7.5, MGAD3 $12.3 \pm 4.5, \mathrm{P}<0.001)$. The decrease in ability to manage their emotions with increasing duration of the disease (MGAD1 15.2 \pm 1.9; MGAD2 12.5 \pm 1.5 ; MGAD3 $4.5 \pm 1.5$; $\mathrm{P}<0.05)$ was found, while the level of empathy was the highest in group of FC of patients with $\mathrm{AD}$ duration from 5 to 8 years (MGAD1 $15.5 \pm 4.5$; MGAD2 $20.0 \pm 2.6$; MGAD3 $12.0 \pm 2.6$; $<0.05$ ), which, presumably indicates a compensatory adaptation of FC to the presence of affective disorder in a family member.

Emotional competence and skills of effective emotional management, which include cognitive, affective and behavioral components, determine system of emotional and rational interpersonal understanding [11]. Social intelligence is considered as an additional factor contributing to effective interpersonal communication, which is the basis for the implementation of empathic-affiliation tendencies. Social intelligence manifests itself in ability to interact with other people, to assess correctly the psycho-emotional state and motivation of communication partners in situations of social interaction, to generalize social information and to predict development of interpersonal interactions. Study of social intelligence level in family caregivers of patients with EMD allowed to reveal certain significant differences in their behavioral and communicative patterns (Table IV). For the psychological testing of FC a full version of social intelligence test by Guildford was used. The results were evaluated in 4 subtests (ST, ST1 "story with completion", ST2 "expression groups", ST3 "non-verbal expression", ST4 "story with addition"), and also composite assessment was done.

Thus, according to ST1 - "story with completion" family caregivers had mediocre abilities in understanding behavior of a patient who is afflicted with PSch or AD under conditions of family functioning (MGPSch $3.22 \pm 0.5$ and MGAD 3.47 \pm 0.9, CG $4.08 \pm 0.5 \mathrm{P}<0.05)$. They could only partially predict further actions of the patient based on the analysis of real situations of family and friendly communication, to understand the feeling of the patient, and on this basis, to determine the effectiveness of communication.

Results for ST1 allow us to conclude that family caregivers of patients with EMD may not always be able to clearly construct a strategy of their own behavior in order to achieve certain goals in treatment of patients. Their behavior may go beyond generally accepted norms, they incorrectly represent the results of their own actions and deeds of others.
According to ST2 - "expression groups", revealed scores allow us to think that family caregivers unlike respondents of CG, experience certain difficulties in assessment states and feelings by nonverbal features in process of interpersonal communication (MGPSch $2.97 \pm 1.1$ and MGAD 3.14 \pm 1.6 , CG $4.18 \pm 0.1, \mathrm{P}<0.001)$. Sensitivity to nonverbal expression greatly enhances the ability to understand others. The ability to read non-verbal signals of another person, to understand them and compare them with verbal ones, is the basis of intuition. When family caregivers of patients with PSch or AD build their behavioral line, they are more likely to focus on the isolated statements of the interlocutor rather than on display of their non-verbal communication. In their understanding, openness and benevolence are not always a guarantee of success in communication, they have reduced sensitivity to emotional states of other people and an incomplete understanding of their own emotional state in conditions of interpersonal interaction, which, undoubtedly, are direct factors that predetermine the dysfunctionality in interpersonal communication.

Performance in ST3 - "verbal expression" (MGPSch $2,8 \pm 0,3$ and MGAD 3,08 $\pm 1,1$, CG 4,13 $\pm 0,7, P<0,001$ ) indicates, that family caregivers, in whose families patients with affective disorders live, have decreased ability to assess the speech of interlocutor quickly and correctly in context of particular situation, to find the right tone for communication, they do not always correctly demonstrate role-based plasticity and understanding of verbal communication meaning. Results obtained in ST4 - "story with addition" (MGPSch 3,1 $\pm 0,7$ and MGAD 3,47 $\pm 0,5$; CG, respectively, $4,49 \pm 0,2, \mathrm{P}<0,001)$, show decreased ability in relatives of patients with EMD to analyze complex situations of interaction in interpersonal communication; they do not always correctly assess the adequacy of the situation for communication and do not fully anticipate how a person will behave in future.

Composite evaluation (MGPSch 3,30 $\pm 2,6$ and MGPAD $3,66 \pm 0,2 ; \mathrm{CG} 4,43 \pm 0,6 ; \mathrm{P}<0,05)$ suggests that adaptive personality resources in family caregivers of patients with EMD have been reduced in interpersonal interaction, they experience difficulty in judgments about interlocutors, are less prone to contact, but more tactic and friendly in communicative interaction in comparison with the control group respondents. They experience difficulties in assessing the needs and intentions of communication, predicting their own behavior and the logic of future events.

In study of the dynamics of social and practical intelligence in family caregivers of patients with EMD, the following patterns were found (Table V): in family caregivers of patients with PSch there was a decrease in social intelligence for all constructs in accordance with the increase in the duration of the disease in a mentally sick family member. This indicates a limited ability to solve social problems, to identify and correctly interpret situations of social interaction, which is probably due to the constant experience of ineffective interpersonal interaction within family system.

Instead, in study of constructs of social intelligence in family caregivers of patients with $\mathrm{AD}$ (Table V) compen- 
satory increase in groups of FC of patients with duration of EMD up to 4 years and from 5 to 8 years (composite assessment MGAD1 2.74 \pm 0.8 ; MGAD2 $3.12 \pm 0.2$; MGAD3 $2.1 \pm 0.5 ; \mathrm{P}<0.001$ ), indicating that $\mathrm{FC}$ are attempting to adapt to the social situation by integrating both positive and negative social experience, attention to the situation context, desire to evaluate social situation objectively.

\section{CONCLUSIONS}

Revealed features should be taken into consideration while developing appropriate psycho-corrective systems for FC of patients with EMD aimed at development and improvement of family interpersonal communication quality in families of patients with EMD, in particular:

1) formation of the ability to predict consequences of social behavior, to understand manifestations of verbal and nonverbal communication, to navigate in the general picture of interpersonal interaction;

2) mastering skills of constructive emotional coping and self-management in conditions of stress caused by mental illness in a close family member;

3) creating conditions for expanding the range of emotional response and developing empathy and reflection in process of interpersonal interaction.

Consequently, family caregivers of patients with schizophrenia and affective disorders demonstrate a decrease in emotional and social intelligence indicators in all subtests, which creates significant obstacles both for effective interpersonal family communication and for the harmonious functioning of a family, in which a mentally sick patient lives, in general. Difficulties of emotional regulation, emotional management, recognition of emotional states of other participants of communication related to the level of emotional and social intelligence of FC are factors, that greatly complicate interpersonal relations in families of patients with EMD and reduce possibilities for psychosocial adaptation of all family members. Revealed features should be taken into consideration when creating appropriate psycho-educational and psycho-corrective programs for family caregivers of patients with endogenous mental disorders.

\section{REFERENCES}

1. Miklowitz DJ. Family Treatment for Bipolar Disorder and Substance Abuse in Late Adolescence. Journal of Clinical Psychology. 2012;68(5):502-513.

2. PshukNG,Stukan, LV, Kaminska, A0. Introducing system of psychotherapeutic intervention for family caregivers of patients with endogenous mental disorders. Wiadomosci Lekarskie. 2018;71(5):980-985.

3. Geriani D, Savithry KSB, Shivakumar S et al. Burden of Care on Caregivers of Schizophrenia Patients: A Correlation to Personality and Coping. Journal of Clinical and Diagnostic Research. 2015;9(3):VC01VC04. doi:10.7860/JCDR/2015/11342.5654.
4. Awad AG, Voruganti LN The burden of schizophrenia on caregivers: a review. Pharmacoeconomics. 2008;26(2):149-162.

5. Ochoa S, Vilaplana M, Haro JM et al. Do needs, symptoms or disability of outpatients with schizophrenia influence family burden? Soc Psychiatry Psychiatr Epidemiol. 2008;43(8): 612-618.

6. Perlick DA, Rosenheck RA, Miklowitz DJ, et al. Caregiver burden and health in bipolar disorder: a cluster analytic approach. J Nerv Ment Dis. 2008;196(6):484-491. doi:10.1097/NMD.0b013e3181773927

7. Gurovich I.Y., Shmukler A.B.,Storozhakova Y.A. Psychosocial therapy and psychosocial rehabilitation in psychiatry. Medpraktika, 2002: 15-25.

8. Bustillo J. The Psychosocial Treatment of Schizophrenia: An Update. The American journal of psychiatry. 2001;158(2):163-175.

9. Gupta S, Isherwood G, Jones K, Van Impe K. Assessing health status in informal schizophrenia caregivers compared with health status in non-caregivers and caregivers of other conditions. BMC Psychiatry. 2015;15:162. Published 2015 Jul 21. doi:10.1186/s12888-015-0547-1

10. Berk L, Berk M, Dodd S et al. Evaluation of the acceptability and usefulness of an information website for caregivers of people with bipolar disorder. BMC Medicine. 2013;11(1):162.

Title of the research work: "Mental disadaptation (clinical phenomenology, mechanisms of formation, complex correction)". State registration number: 0115 U004000. Topic Code: 616.891-08. Venue: National Pirogov Memorial Medical University, Vinnytsia, Ukraine. Timeframe: 01/01/2015 31/12/2019

\section{ORCID and contributionship:}

Anna O. Kaminska - 0000-0003-3281-989X A,B,D,F

Nataliia G. Pshuk - 0000-0001-9857-2565 A,E

Yuliana Y. Martynova - 0000-0002-7372-8743 B,C

\section{Conflict of interest:}

The Authors declare no conflict of interest

\author{
CORRESPONDING AUTHOR \\ Anna 0. Kaminska \\ Shevchenka street, 71a, Vinnytsya, Ukraine \\ tel: +380979719887 \\ e-mail: adonidisvernalis@gmail.com
}

Received: 18.04 .2019

Accepted: 12.11.2019

A - Work concept and design, B - Data collection and analysis, C - Responsibility for statistical analysis, D-Writing the article, $\mathbf{E}$-Critical review, $\mathbf{F}$ - Final approval of the article 